factors have been associated with the presence of HTPR in patients with CAD and PAD, including CYP2C19 loss of function polymorphism, drug-drug interactions, and medical comorbidities. Gender differences are another factor that might influence the levels of platelet inhibition while on Clopidogrel and hence, HTPR. Differences by Gender exist in platelet biology, count, and activation. The evidence for the influence of Gender in HTPR is limited, but a possible association has been described. In this study, we described the association of Gender with HTPR and Major Adverse Cardiovascular Events (MACEs) occurrence. The data is from a sample of HispanoCaribbean patients on Clopidogrel therapy alone or in combination with Aspirin that were retrospectively evaluated from an ongoing trial in Puerto Rico. The result of this study provided evidence of the influence that Gender has on antiplatelet therapy function and MACEs occurrence. METHODS/STUDY POPULATION: The population in the study consisted of Hispano-Caribbean patients using Clopidogrel alone or in combination with Aspirin for coronary artery disease, peripheral arterial disease, or cerebrovascular disease. The sample was obtained from multiple hospital institutions with cardiovascular services in Puerto Rico during the years 2016-2019. Patients were part of the ongoing trial, "Adopting a precision medicine paradigm in Puerto Rico: leveraging ancestral diversity to identify predictors of Clopidogrel response in Caribbean Hispanics." The sample size consisted of 150 patients. Participants were recruited during routine medical care, pre-admission evaluation for elective cardiac procedures, or during hospitalization in the participating institutions. Platelet reactivity testing was performed with the system Verify Now ${ }^{\circledR}$ to determine PRU values, and High on-treatment platelet reactivity was defined as $P R U \geq 208$. One year after recruitment, the patients were re-evaluated for the occurrence of MACEs. The association of the variables HTPR, occurrence of MACEs, and Gender were assessed using logistic regression in addition to the role of HTPR and Gender for predicting MACE occurrence. The analysis was done using the statistic software Intellectus ( ). RESULTS/ ANTICIPATED RESULTS: The sample consisted of 67 females and 83 males with and Mean age of 67.87 years and 61.11 years, respectively. The prevalence of HTPR in the sample was $32.67 \%$ (n $=49)$ with $36 \%(n=24)$ for females, and $30 \%(n=25)$ for males. The mean PRU values were 179.54 for females and 170.81 for males. The percentage of MACEs one year after recruitment was $29.33 \%$ (n $=44)$ with $43 \%$ on females $(n=19)$, and $57 \%$ on males $(n=25)$. Logistic regression for Gender predicting HTPR was non-significant with a $\chi 2(2)=0.55, \mathrm{p}=.758$, and McFadden $\mathrm{R}^{2}=0.00$. Also, logistic regression for the effects of Gender and HTPR on the Odds of MACEs occurrence was not significant based on a model with an alpha of $0.05, \chi 2(2)=1.99, \mathrm{p}=.370$, and McFadden $\mathrm{R}^{2}=0.01$. DISCUSSION/SIGNIFICANCE OF IMPACT: The sample consisted of 67 females and 83 males with and Mean age of 67.87 years and 61.11 years, respectively. The prevalence of HTPR in the sample was $32.67 \%(n=49)$ with $36 \%(n=24)$ for females, and $30 \%(n=25)$ for males. The mean PRU values were $179.54( \pm 70.42)$ for females and 170.81( \pm 64.89$)$ for males. The percentage of MACEs one year after recruitment was $29.33 \%(n=44)$ with $43 \%$ on females $(n=19)$, and $57 \%$ on males $(n=25)$. Logistic regression for Gender predicting HTPR was non-significant with a $\chi 2(2)=0.55, p=.758$, and McFadden $R^{2}=0.00$. Also, logistic regression for the effects of Gender and HTPR on the odds of MACEs occurrence was not significant based on a model with an alpha of $0.05, \chi 2(2)=1.99$, $\mathrm{p}=.370$, and McFadden $\mathrm{R}^{2}=0.01$.
Metabolomic Identifiers Predictive of Adverse Events due to Acetaminophen Administration

Brandon Joseph Sonn, PhD Candidate ${ }^{1}$, Kennon Heard ${ }^{1}$, and Andrew Monte ${ }^{1}$

${ }^{1}$ University of Colorado at Denver

OBJECTIVES/GOALS: Acetaminophen (Tylenol, APAP) toxicity has been well documented and well explored over the last 50 years. However, there has been no investigation into identification of specific metabolites that can predict which patients will have adverse reactions to therapeutic doses of APAP. METHODS/STUDY POPULATION: 205 subjects recruited from the Denver, CO community received the highest recommended daily dosing of APAP, 4 grams, for 16 days. Subjects were grouped by 1) alanine aminotransferase (ALT) at any monitored time point above 60units/L (n $=20)$ vs 2 ) no increase in ALT at any time point $(n=185)$. Blood was collected at days $0,4,7,16$, and 31 . Samples were run on ultra-high performance liquid chromatography mass spectrometry with 27 heavy-labeled standards for metabolites documented to be associated with APAP metabolism. Data will be analyzed to look for significant changes in metabolite and demographic variable expressions using t-tests, chi square and logistic regression, as appropriate. RESULTS/ANTICIPATED RESULTS: It is expected that there will be greater elevations of conjugated non-toxic APAP metabolites (APAP-glucuronide, APAP-sulfate) in subjects whose ALT did not elevate because of successful hepatoprotection. Conjugated APAP metabolites are expected to only be present in samples taken after APAP therapy initiation confirming exposure as compared to being predictive of toxic response. Increases in lactate and cysteine in pre-exposure samples would allow for prediction of APAP toxicity as they are expected to have increased expression in subjects whose ALT became elevated which is indicative of increased hepatic damage due to oxidative damage. DISCUSSION/ SIGNIFICANCE OF IMPACT: Identification of metabolites and/ or demographic factors associated with toxic response to APAP prior to administration could advise APAP recommendations. Quantification of post-APAP administration metabolites would identify extent of successful hepatoprotective mechanisms.

\section{Noninvasive hybrid ultrasound and photoacoustic imaging for the assessment of liver fibrosis}

Laith Riyadh Sultan ${ }^{1}$, Mrigendra Karmacharya ${ }^{1}$, Julia D'Souza ${ }^{1}$, Brooke Kirkham ${ }^{1}$, Angela K Brice ${ }^{1}$, Andrew KW Wood ${ }^{1}$, Stephen Hunt $^{1}$, and Chandra Sehgal ${ }^{1}$

${ }^{1}$ University of Pennsylvania School of Medicine

OBJECTIVES/GOALS: The detection of liver fibrotic changes at an early and reversible stage is essential to prevent its progression to end-stage cirrhosis and hepatocellular carcinoma. Liver biopsy, which is the current gold standard for fibrosis assessment, is accompanied by several complications due to its invasive nature in addition to sampling errors and reader variability. In this study, we evaluate the use of quantitative parameters extracted from hybrid ultrasound and photoacoustic imaging to detect and monitor fibrotic changes in 
a DEN rat model. METHODS/STUDY POPULATION: Liver fibrotic changes were induced in 34 Wistar male rats by oral administration of Diethylnitrosamine (DEN) for 12 weeks. 22 rats were imaged with B-mode ultrasound at 3 different time points (baseline, 10 weeks and 13 weeks) for monitoring liver texture changes. Texture features studied included tissue echointensity (liver brightness normalized to kidney brightness) and tissue heterogeneity. 12 rats were imaged with photoacoustic imaging at 4 time points (baseline, $5 \mathrm{wks}$, $10 \mathrm{wks}$, and $13 \mathrm{wks}$ ) to look at changes in tissue oxygenation. Hemoglobin oxygen saturation (sO2A) and hemoglobin concentration $(\mathrm{HbT})$ in the right and left lobes of the liver were measured. 8 rats were used as controls. Liver tissue samples were obtained following 13 weeks from DEN start time for METAVIR histopathology staging of fibrosis. RESULTS/ANTICIPATED RESULTS: Texture features studied showed an increase with time in DEN rats. Normalized echointensity increased from $0.28 \pm 0.06$ at baseline to $0.46 \pm 0.10$ at 10 weeks $(p<0.0005)$ and $0.53 \pm 0.15$ at 13 weeks in DEN rats $(p<0.0005)$. In the control rats, echointensity remained at an average of $0.25 \pm 0.05(p=0.31)$. Tissue heterogeneity increased over time in the DEN-exposed rats from a baseline of $208.7 \pm 58.3$ to $344.6 \pm 52.9$ at 10 weeks $(p<0.0005)$ and $376.8 \pm 54.9$ at 13 weeks $(p=0.06)$ however it stayed constant at $225.7 \pm 37.6$ in control rats $(p=0.58)$. The quantitative analyses of the photoacoustic signals showed that blood oxygen saturation significantly increased with time. At 5 weeks sO2AvT increased by $53.83 \%( \pm 0.25)$, and $\mathrm{HbT}$ by $35.31 \%( \pm 0.07)$. Following 10 weeks of DEN; sO2AvT by $92.04 \%( \pm 0.29)$, and HbT by $55.24 \%( \pm 0.1)$. All increases were significant $p<0.05$. In the 13 th week, however, the values of all of these parameters were lower than those in the 10th week, however, the decrease was statistically insignificant. DISCUSSION/ SIGNIFICANCE OF IMPACT: Quantitative features from B-mode ultrasound and photoacoustic imaging consistently increased over time corresponding to hepatic damage, inflammation and fibrosis progressed. The use of this hybrid imaging method in clinical practice can help meet the significant need for noninvasive assessment of liver fibrosis.

4167

Peri-transplant Lung Microbiome Reveal Oral Bacteria, Pepsin And Inflammatory Markers Co-associate With Primary Graft Dysfunction, Implicating Aspiration As A Potential Contributor

John Evan McGinniss ${ }^{1}$, Joshua M. Diamond ${ }^{1}$, Melanie C. Brown ${ }^{1}$, Ed Cantu $^{1}$, Jason D. Christie ${ }^{1}$, Rick D. Bushman ${ }^{1}$, and Ronald G. Collman ${ }^{1}$

${ }^{1}$ University of Pennsylvania School of Medicine

OBJECTIVES/GOALS: Primary graft dysfunction (PGD) is acute lung injury in the first three days after lung transplant. Patients that experience PGD have increased mortality and an increased risk of chronic lung allograft dysfunction. The pathogenesis is thought to be an ischemia-reperfusion injury but is incompletely understood and there are no specific therapies. We investigated the role of the microbiome in PGD and associations with inflammation and markers of aspiration. METHODS/STUDY POPULATION: We collected airway lavage samples from lung transplant donors before procurement and recipients after reperfusion. We extracted DNA, amplified the bacterial $16 \mathrm{~S}$ rRNA gene, and sequenced on the Illumina MiSeq platform. QIIME2 and Deblur were used for bioinformatic analysis. R packages were used for downstream analysis and visualizations. The host response was quantified using the Milipore 41-plex Luminex and an
ELISA for pepsin. Clinical data was collected by the Penn Lung Transplant Outcomes Group. PGD was assessed by degree of hypoxemia and chest X-ray findings in the 72 hours after transplant. RESULTS/ANTICIPATED RESULTS: There was no significant difference in alpha diversity (Shannon index, $\mathrm{p}=0.51$ ), biomass (via comparison of $16 \mathrm{~S}$ amplicon PicoGreen, $\mathrm{p}=0.6$ ), or beta diversity (Weighted UniFrac, $\mathrm{p}=0.472$, PERMANOVA) between subjects with PGD grade $3(n=36)$ and those that did not $(n=96)$. On taxonomic analysis, we found an enrichment of Prevotella in donor and recipient lungs that went on to develop PGD $(p=0.05)$. To follow up this finding we measured immune response and pepsin concentrations in recipient lungs. We found elevated levels in 35/41 cytokines measured in subjects that developed PGD as well as an elevation in pepsin and a correlation between pepsin concentration and Prevotella relative abundance (Figure 1). Additionally, Prevotella relative abundance had statistically significant positive correlations with multiple cytokines such as IL-6 (Pearson's $=0.26, \quad \mathrm{p}=0.009$ ) and eotaxin (Pearson's $=0.24, \mathrm{p}$ $=0.016)$. DISCUSSION/SIGNIFICANCE OF IMPACT: There is an enrichment of oral anerobes in lung allografts that eventually develop PGD. This is associated with elevated levels of pepsin and markers of inflammation. These lines of evidence suggest aspiration contributes to priming the allograft for PGD.

\section{6}

\section{Personalizing Care For Colorectal Cancer: Identifying Novel Opportunities}

Zachary Rivers ${ }^{1}$, David Stenehjem², Emil Lou ${ }^{2}$, Andrew Nelson², Pamala Jacobson ${ }^{2}$, and Karen Kuntz ${ }^{2}$

${ }^{1}$ University of Minnesota CTSI; ${ }^{2}$ University of Minnesota

OBJECTIVES/GOALS: This project seeks to understand how personalized medicine can optimize care for patients with colorectal cancer. It identifies opportunities for personalized medicine to improve clinical outcomes, and uses cost-effectiveness analysis to assess the clinical and financial impact of this approach. METHODS/STUDY POPULATION: This project uses two methods to understand the impact of personalized medicine. First, this project has used SEER-Medicare data in conjunction with Clinical Pharmacogenetics Implementation Consortium guidelines to identify medications used by patients with colorectal cancer that can be impacted by genetic variants. This data will then be combined with population genetic variant rates to understand the likely impact screening for a given variant will have on medication response and adverse events. Medication use frequencies and genetic variant rates are then used to populate cost-effectiveness models that simulate the clinical and financial outcomes, identifying optimal genes to screen. RESULTS/ANTICIPATED RESULTS: The first result will be a comprehensive overview of treatment patterns for patients with colorectal cancer in the United States, as well as the treatments used for disease-induced comorbidities. The second result will be the identification of genetic variants based on population rates and medication utilization that should be screened in this patient population. The final result will be a breakdown of the clinical and financial outcomes associated with implementing screening for the identified genes. Preliminary results from a two-gene cost-effectiveness analysis demonstrates that screening for variants in those genes improves both clinical and financial outcomes. DISCUSSION/ SIGNIFICANCE OF IMPACT: This project demonstrates how current treatment approaches can be optimized via personalized medicine. It uses epidemiological methods to identify opportunities to integrate genetic findings from other diseases, and uses cost- 